\title{
L'énigme des guides de pratique clinique
}

\author{
par Clarence Chant
}

$\mathrm{L}$ 'Institute of Medicine définit les guides de pratique clinique (GPC) comme " des énoncés élaborés de façon systématique pour assister les praticiens et les patients dans la prise de décisions en matière de soins de santé dans une situation clinique définie ". ${ }^{1}$ Les prétendus avantages des GPC sont notamment l'amélioration des résultats cliniques, la standardisation de la pratique en assurant un certain niveau minimum de soins et en dissuadant le recours aux soins non fondés sur des données probantes, l'amélioration de l'uniformité et de l'efficience des soins, la découverte des manques de connaissances, la stimulation des discussions entre professionnels et la réduction des coûts des soins. ${ }^{2}$ En revanche, la littérature ne fait pas clairement état des avantages réels tirés de la mise en pratique de tels GPC. Nonobstant les avantages potentiels des GPC, la mise en œuvre de ces derniers est un processus complexe et exigeant. D'ailleurs, une récente étude de grande envergure, comparative, à répartition aléatoire par grappes a révélé que les interventions d'application des connaissances exhaustives et à multiples facettes joignant jusqu’à $96 \%$ du public cible de diverses unités de soins intensifs n'avaient entraîné qu'une augmentation de $8 \%$ sur 2 ans du respect d'une série de recommandations factuelles formulées dans un guide de pratique sur la pneumonie sous ventilation assistée. ${ }^{3}$ Parallèlement, les cliniciens ont formulé plusieurs inquiétudes quant aux GPC, dont une piètre adoption par les praticiens, un risque de substitution au jugement clinique, un obstacle à l'enseignement et à la curiosité, et leur emploi inapproprié à des fins juridiques et de couverture d'assurance.

Dans ce numéro du Journal canadien de la pharmacie hospitalière (JCPH), les auteurs de la chronique « Le pour et le contre " débattent s'il ne faudrait pas que les normes d'établissement ou d'agrément exigent que les praticiens cliniciens consignent la raison pour laquelle ils choisissent de ne pas adhérer à des guides de pratique clinique largement reconnus., Les auteurs des deux camps présentent de nombreux arguments valables étayant leur raisonnement. L'un des points soulevés questionnant la mesure dans laquelle les guides de pratique sont actuellement fondés sur des données probantes mérite qu’on creuse le sujet. ${ }^{5}$ En fait, je crois que les données probantes sur lesquelles reposent ces guides sont essentielles, car les soins factuels sont considérés comme la norme de pratique actuelle des sciences de la santé et sont certainement un aspect que nous, pharmaciens cliniciens, essayons tous très fort d'inculquer à nos étudiants, à nos collègues praticiens et même à nos administrateurs. Par conséquent, avant d'envisager s'il faudrait exiger des praticiens qu'ils motivent les raisons pour lesquelles ils choisissent de ne pas adhérer aux GPC, il faut se demander si les GPC eux-mêmes ont été développés selon des méthodes rigoureuses et si leur utilisation est valable.

La réponse est, du moins à mes yeux, quelque peu inattendue. D'abord, il existe de nombreux outils validés pour évaluer de façon critique les GPC. ${ }^{6}$ Un de ces outils, AGREE (Appraisal of Guidelines for Research and Evaluation), ${ }^{7}$ est en fait celui qu'utilise la Société canadienne des pharmaciens d'hôpitaux lorsqu'on lui demande d'avaliser des GPC développés à l'externe. Or, l'évaluation de GPC à l'aide d'un outil validé ne fait pas systématiquement partie des programmes de pharmacie, pas plus qu'il n'y a de publications qui s'attardent à l'évaluation critique des GPC, comme ce qui existe pour les études comparatives à répartition aléatoire (p. ex., ACP Journal Club, publié par l'American College of Physicians). Dans une étude, lorsque les outils d'évaluation des GPC ont été utilisés pour faire l'évaluation critique des guides actuels pour les soins intensifs, les scores moyens de qualité obtenus étaient au mieux de $50 \%$ !8 Le problème n'est pas unique aux soins intensifs et a été confirmé dans plusieurs autres secteurs de la médecine. ${ }^{9}$ Heureusement, la qualité des GPC s'améliore avec le temps. ${ }^{8}$ En outre, d'autres moyens, comme le système de recommandations GRADE (Grading of Recommendations Assessment, Development and Evaluation $)^{10}$ et un cadre normatif proposé pour la formulation $^{11}$, sont des améliorations bienvenues pour améliorer la qualité des GPC. Le système de recommandations GRADE est particulièrement utile, car le niveau de recommandation final obtenu tient compte du point de vue des patients, de la qualité de vie et du rapport coût-efficacité. Cela est particulièrement utile dans des domaines comme la gériatrie, où les praticiens doivent quelques fois s'éloigner de façon appropriée des recommandations formulées dans les guides en tenant compte de facteurs particuliers aux patients. En dernier lieu, l'Institute 
of Medicine a récemment publié ses propres normes de développement de GPC fiables. ${ }^{12}$

Étant donné la profusion des GPC et leur emploi potentiel dans des documents officiels ayant des implications juridiques, comme illustré par le débat dans le présent numéro du JCPH, j'inviterais tous les étudiants en pharmacie et les pharmaciens (ou, mieux encore, tous les professionnels de la santé) à évaluer de façon critique les GPC à l'aide d'un outil validé, tout comme on le ferait pour une étude comparative à répartition aléatoire, avant de décider d'en adopter ou non les recommandations. Après tout, il y a des guides pour les guides!

\section{Références}

[Traduction par l'éditeur]

1. Field MJ, Lohr KN, editors; Institute of Medicine, Committee to Advise the Public Health Service on Clinical Practice Guidelines. Clinical practice guidelines: directions for a new program. Washington (DC): National Academy Press; 1990.

2. Woolf SH, Grol R, Hutchinson A, Eccles M, Grimshaw J. Clinical guidelines: potential benefits, limitations, and harms of clinical guidelines. BMJ. 1999;318(7182):527-30.

3. SinuffT, Muscedere J, Cook DJ, Dodek PM, Anderson W, Keenan SP, et al., for Canadian Critical Care Trials Group. Implementation of clinical practice guidelines for ventilator-associated pneumonia: a multicenter prospective study. Crit Care Med. 2013;41(1):15-23.

4. Wilbur K. Should clinical practitioners, as part of institutional or accreditation standards, be required to document rationale when they choose to not adhere to widely accepted clinical practice guidelines? The "Pro" side. Can J Hosp Pharm. 2013;66(4):253-4.

5. Wilby K. Should clinical practitioners, as part of institutional or accreditation standards, be required to document rationale when they choose to not adhere to widely accepted clinical practice guidelines? The "Con" side. Can J Hosp Pharm. 2013;66(4):254-5.

6. Vlayen J, Aertgeerts B, Hannes K, Sermeus W, Ramaekers D. A systematic review of appraisal tools for clinical practice guidelines: multiple similarities and one common deficit. Int J Qual Health Care. 2005;17(3):235-42.
7. AGREE collaboration. Development and validation of an international appraisal instrument for assessing the quality of clinical practice guidelines: the AGREE project. Qual Saf Health Care. 2003;12(1):18-23.

8. Sinuff T, Patel RV, Adhikari NK, Meade MO, Schünemann HJ, Cook DJ. Quality of professional society guidelines and consensus conference statements in critical care. Crit Care Med. 2008;36(4):1049-58.

9. Shaneyfelt TM, Mayo-Smith MF, Rothwangl J. Are guidelines following guidelines? The methodological quality of clinical practice guidelines in the peer-reviewed medical literature. JAMA. 1999;281(10):1900-5.

10. Atkins D, Best D, Briss PA, Eccles M, Falck-Ytter Y, Flottorp S, et al; GRADE Working Group. Grading quality of evidence and strength of recommendations. BMJ. 2004;328(7454):1490.

11. Shiffman RN, Shekelle P, Overhage JM, Slutsky J, Grimshaw J, Deshpande AM. Standardized reporting of clinical practice guidelines: a proposal from the Conference on Guideline Standardization. Ann Intern Med. 2003;139(6):493-8.

12. Standards for developing trustworthy clinical practice guidelines. Washington (DC): Institute of Medicine of the National Academies; 2011. Publié au www.iom.edu/Reports/2011/Clinical-Practice-Guidelines-We-CanTrust/Standards.aspx. Consulté le 10 mai 2013.

Clarence Chant, Pharm. D., BCPS, FCSHP, FCCP, est chef de pharmacie au St Michael's Hospital, à Toronto, en Ontario. ॥ est également rédacteur adjoint du $J C P H$.

Intérêts concurrents : aucun déclaré.

Adresse de correspondance :

Dr Clarence Chant

Pharmacy Department

St Michael's Hospital

30 Bond Street, Room B10007

Toronto ON M5B 1W8

Courriel : CHANTC@smh.ca 